

\begin{tabular}{|c|l|}
\hline Title & $\begin{array}{l}\text { Toward automatic classification of partial } \\
\text { discharge sources with neural networks }\end{array}$ \\
\hline Author(s) & $\begin{array}{l}\text { Hirata, Akimasa; Nakata, Syou; Kawasaki, Zen- } \\
\text { ichiro }\end{array}$ \\
\hline Citation & $\begin{array}{l}\text { IEEE Transactions on Power Delivery. 21(1) } \\
\text { p. 526-p. 527 }\end{array}$ \\
\hline Issue Date & $2006-01$ \\
\hline oaire:version & VoR \\
\hline URL & $\begin{array}{l}\text { https://hdl.handle. net/11094/3459 } \\
\text { O2006 IEEE. Personal use of this material is } \\
\text { permitted. However, permission to } \\
\text { reprint/republish this material for advertising } \\
\text { or promotional purposes or for creat ing new } \\
\text { collective works for resale or redistribution } \\
\text { to servers or lists, or to reuse any } \\
\text { copyrighted component of this work in other } \\
\text { works must be obtained from the IEEE.. }\end{array}$ \\
\hline rights \\
\hline Note & \begin{tabular}{l} 
\\
\hline
\end{tabular} \\
\hline
\end{tabular}

Osaka University Knowledge Archive : OUKA

https://ir. Library. osaka-u. ac. jp/

Osaka University 


\title{
Toward Automatic Classification of Partial Discharge Sources With Neural Networks
}

\author{
Akimasa Hirata, Member, IEEE, Syou Nakata, and Zen-Ichiro Kawasaki, Member, IEEE
}

\begin{abstract}
In this letter, we propose an automatic classification scheme of discharge sources on the basis of Fourier spectrum for received electromagnetic $(\mathrm{EM})$ pulses. Confirming the reproducibility of EM pulses from each source validates this scheme. The recognition rate for six discharge sources is $80 \%$ or better. The feature of our system is time efficient and to treat hundreds of pulses.
\end{abstract}

Index Terms-Digital systems, fault diagnosis, neural-network applications, partial discharges.

\section{INTRODUCTION}

$\mathbf{P}$ ARTIAL-DISCHARGE (PD) activity is a symptom of an insulation breakdown or a warning for the insulation failure of electrical power apparatus and can emit impulsive electromagnetic (EM) noise. For realizing the high reliability of power systems, it is desirable to identify the location of PD sources with high accuracy. Our group has proposed remote-sensing techniques on the basis of the time difference of received pulses [1] and multiple signal classification (MUSIC) [2]. Note that another group has carried out a similar approach [3]. According to this literature, the remote sensing techniques for the PD source localization are found to be effective, but the error in estimated source location is not always small. Thus, another approach will also be required as an auxiliary scheme for source detections.

It has been reported that waveforms emitted from PD sources are different from each other [4]. It is not easy to classify them by a slight look at time-domain waveforms. Also, the waveform of an EM wave is distorted by the reflection from the ground. However, this affects the frequency spectrum of an EM wave marginally. Thus, let us pay attention to the frequency spectrum of EM pulses emitted from PD sources, since they are largely dependent on power-line defects [4], [5]. Then, we proposed to apply neural networks to this classification [6], particularly to realize an automatic system. Similar approaches have been reported for online PD measurement systems [7], [8]. In this letter, first, we confirm the reproducibility of EM pulses from PD sources in order to ensure the validity of our proposal. Then, we demonstrate the effectiveness of the application of neural networks to the automatic classification of six discharge sources.

Manuscript received August 10, 2004; revised January 4, 2005. This work was supported in part by the Kansai Research Foundation for Technology Promotion, Japan. Paper no. PESL-00074-2004.

A. Hirata is with the Nagoya Institute of Technology, Nagoya 466-8555, Japan.

S. Nakota and Z. Kawasaki are with the Department of Communications Engineering, Osaka University, Osaka 565-0871, Japan (e-mail: ahirata@ieee.org).

Digital Object Identifier 10.1109/TPWRD.2005.848439

\section{EXPERIMENTAL SETUP AND Signal PROCESSING}

\section{A. Experimental Setup}

Only the outline of our measurement system is described, since it can be found in [1]. Wide-band antenna array is used as an EM wave sensor and located at $5-15 \mathrm{~m}$ from a discharge source. The antenna is connected to a digital storage oscilloscope (DSO) by a coaxial cable through a band-pass filter $(25-250 \mathrm{MHz})$. The EM waves are digitized at a sampling rate of $500 \mathrm{MHz}$ by the DSO, which is controlled by a personal computer (PC). In order to detect an EM pulse train, the memory of the DSO is divided into 1000 segments. Each segment records a pulse for the time window of $2.0 \mu \mathrm{s}$.

The total of six discharge sources is considered: three simulated discharge sources and actual PD sources in motors of three electrical power apparatus. The former sources are a needleplane electrode, a faulty contact of two strain insulators, and a cracked pin-type insulator. The latter sources are PDs in the insulators of electrical power apparatus: forced draft fans, cooling water pumps, and condensate booster pumps.

\section{B. Signal Processing}

1) Correlation: The reproducibility of EM pulses from PD sources is investigated. The maximum value of a cross correlation coefficient between an ensemble average of EM pulses and each EM pulse are calculated for the sources. Note that the ensemble-averaged waveform for a source is calculated by averaging more than a thousand pulses. The reproducibility of EM pulses from a specific discharge source is evaluated with the average value of a maximum cross-correlation coefficient. This is evaluated in the time domain for fixed source-antenna distance. One of the waveforms with the highest signal-to-noise ratio (SNR) received by the antennas is used for this evaluation.

2) Neural Networks: Neural networks are one of the candidates to realize an automatic classification, because of their capability of pattern recognition [9]. A structure of three-layered networks is chosen in this paper, because of its simplicity. A back-propagation algorithm is applied to train the networks with a learning rate of 0.2 and a momentum rate of 0.9 . These values are chosen so that the network is learned efficiently.

To recognize the spectrum patterns by the neural network, it is important to select a set of input data properly. Our procedure for producing the learning signals for the neural network is as follows. The waveforms in the time domain are transformed into the Fourier spectrum. Then, the Fourier spectrum of the wave is normalized so that the total of the Fourier spectrum becomes unity. Further, mean values for the typical spectrum are considered as learning signals. 


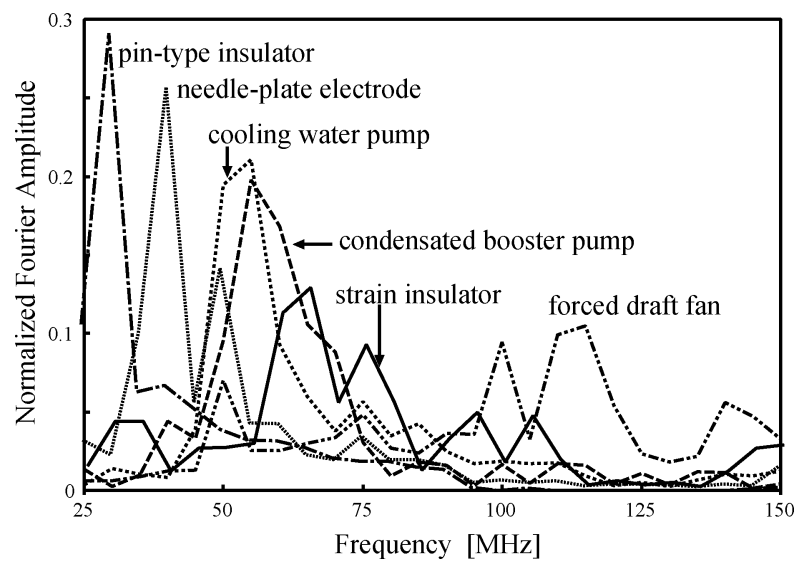

Fig. 1. Normalized Fourier amplitude for different discharge sources.

\section{RESULTS}

Cross correlations of EM pulses from PD sources are evaluated only for needle-plane electrode and a motor in a forced draft fan, in order to avoid repetition. The cross correlation between pulses emitted from them were 0.988 and 0.752 . Note that the latter value is somewhat smaller than the former. This is because the experiment has been conducted in a power plant and, thus, the background noise was not negligible. Also, note that the gap between the needle and plane was varied from 1.0 to $5.0 \mathrm{~mm}$. However, no clear difference was observed between the spectrums. From these results, not the magnitude of capacity of discharges but the kind of sources is essential to characterize the frequency spectrum of EM pulses emitted from discharges.

In [1], we have shown that the frequency spectrum for discharge sources is concentrated in the frequency range below $150 \mathrm{MHz}$. In order to learn neural networks, we decide to pick out a total of 26 spectral components from statistical fast Fourier transform (FFT) results. That is, there is the frequency band of $25-150 \mathrm{MHz}$ with an interval of $5 \mathrm{MHz}$. Therefore, the neural network is designed to have 26 inputs, 10-50 hidden units, and six output neurons. Fig. 1 shows the comparison of the normalized spectrum between discharge sources. As is evident from this figure, significant differences can be observed between them. It is worth noting that the spectrum of a cooling water pump and condensate booster pump are similar. The reason for this would be the analogy of their inner structure, together with their similar functions. Therefore, they are treated as the same source and, thus, the number of output neurons becomes five.

Table I shows the recognition results by neural networks with a different number of hidden units. Note that measurements have been conducted in the situation where different sources exist. The relative positions of antennas are changed in order to enhance the validity. Also, note that they are classified by DOA estimation [1]. From this table, it is found that the noise sources can be reasonably classified. The recognition rate was $80 \%$ or better. It is worth noting that the recognition rate is not so affected by the number of hidden units. This recognition rate is sufficient as we have treated not one pulse but a train with hun-
TABLE I

RECOGNITION RATIO OF Discharge SOURCES BY THE NEURAL NETWORKS (1: NeEdle-Plane Electrode, 2: Strain Insulator, 3: Pin-Type INSULATOR, 4: FORCED DRAFT FAN, AND 5: COOLING BOOSTER PUMP/CONDENSATED BOOSTER PUMP)

\begin{tabular}{l|c|c|c|c|c}
\hline \multirow{2}{*}{$\begin{array}{l}\text { No. of } \\
\text { Hidden Units }\end{array}$} & \multicolumn{5}{|c}{ Recognition rate (\%) } \\
\cline { 2 - 6 } & 1 & 2 & 3 & 4 & 5 \\
\hline 10 & 96.5 & 99.0 & 93.0 & 83.0 & 95.0 \\
\hline 20 & 98.0 & 100.0 & 82.0 & 85.0 & 86.5 \\
\hline 30 & 98.0 & 100.0 & 92.5 & 82.5 & 89.5 \\
\hline
\end{tabular}

dreds of pulses and, thus, a computationally efficient algorithm proposed in this system can be practical. Also, in this system, it is not difficult to improve this recognition rate by using fine frequency resolution or using a wavelet transformation to de-noise the received signals (e.g., it should be noted that sophisticated signal processing, which is a combination of Welch and MUSIC algorithms, is proposed to extract more exact frequency spectrum of EM waves emitted from PD sources [6]. The scheme can be useful to know the frequency characteristics of EM waves. However, it is not computationally efficient and, thus, is not so suitable to our approach, which treats hundreds of pulses.

\section{SUMMARY}

In this letter, we proposed an automatic classification scheme of PD sources on the basis of Fourier spectrum. Confirming the reproducibility of EM pulses from each source validated this scheme. The recognition rate was $80 \%$ or better for six sources. This scheme can be extended for any number of sources whose spectrum is known. Another feature of our system is to treat hundreds of pulses and time efficiency.

\section{REFERENCES}

[1] M. Soliman, A. Hirata, T. Morimoto, and Z. Kawasaki, "Numerical and experimental study on three-dimensional localization for ultra-wideband impulsive noise sources," J. Electromagn. Waves Appl., vol. 19, no. 1, pp. 141-153, Jan. 2005

[2] A. Hirata, T. Morimoto, and Z. Kawasaki, "DOA estimation of ultrawideband EM waves with MUSIC and interferometry," IEEE Wireless Propagat. Lett., vol. 3, pp. 190-193, 2003.

[3] C. H. Peck and P. J. Moore, "A direction-finding technique for wideband impulsive noise source," IEEE Trans. Electromagn. Compat., vol. 43, no. 2, pp. 149-154, May 2001.

[4] A. Tungkanawanich, Z. Kawasaki, and K. Matsuura, "Analysis of VHFwideband impulsive electromagnetic noises on power distribution lines," Trans. Inst. Elect. Eng. Jpn., vol. 120-B, no. 11, pp. 1538-1544, 2001.

[5] I. Portugues, P. J. Moore, and A. Glover, "Frequency domain characterization of partial discharges via a noninvasive measurement system," in Proc. Int. Conf. Properties Applications Dielectric Material, vol. 3, 2003, pp. 835-838.

[6] S. Nakata, M. S. Soliman, T. Morimoto, A. Hirata, and Z. Kawasaki, "Proposal of detection system for partial discharge sources," in Proc. Int. Conf. Insulation Condition Monitoring Electr. Plant, vol. B-05, 2003.

[7] H. J. van Breen et al., "Standardization of on-line VHFPD measurements on turbo generators," IEEE Trans. Dielect. Elect. Insul., vol. 9, no. 1, pp. 140-149, Feb. 2002.

[8] A. Contin, A. Cavallini, G. C. Montanari, G. Pasini, and F. Puletti, "Digital detection and fuzzy classification of partial discharge signals," IEEE Trans. Dielect. Elect. Insul., vol. 9, no. 3, pp. 335-348, Jun. 2002.

[9] D. E. Rumelhart and J. L. McClelland, Parallel Distribution Processing. Cambridge, MA: MIT Press, 1986. 